\title{
Dense laser-driven electron sheets as relativistic mirrors for coherent production of brilliant $\mathrm{X}$-ray and $\boldsymbol{\gamma}$-ray beams
}

\author{
D. Habs • M. Hegelich • J. Schreiber • M. Gross • \\ A. Henig • D. Kiefer • D. Jung
}

Received: 8 August 2008 / Published online: 19 October 2008

(c) The Author(s) 2008. This article is published with open access at Springerlink.com

\begin{abstract}
Several techniques exist to obtain brilliant X-ray beams by coherent reflection from relativistic electrons $\left(E_{\mathrm{e}}=\gamma m c^{2}\right)$ with Doppler frequency upshift of $4 \gamma^{2}$. We describe a new approach starting with an ultra-thin solid target. Larger 'driver'-laser intensities with high contrast are required to produce dense electron sheets. Their acceleration in vacuum results in a transverse momentum component besides the dominant longitudinal momentum component. The counter-propagating 'production' laser for optimum Doppler boost in X-ray production by reflection has to be injected opposite to the electron direction and not opposite to the driver laser. Different measures to increase the reflectivity of the electron sheet via laser trapping or freeelectron-laser-like micro-bunching are discussed, extending the photon energy into the $\mathrm{MeV}$ range. Here, first-order estimates are given.
\end{abstract}

PACS 42.55.Vc $\cdot 29.27 .-\mathrm{a} \cdot 41.75 . \mathrm{Jv} \cdot 41.75 . \mathrm{Ht}$

\section{Introduction}

Compact, intense, brilliant X-ray sources have a broad spectrum of applications from single-molecule imaging to diag-

D. Habs (凶) · J. Schreiber · M. Gross · A. Henig · D. Kiefer · D. Jung

Faculty of Physics, Ludwig-Maximilians-University München, 85748 Garching, Germany

e-mail: Dietrich.Habs@physik.uni-muenchen.de

D. Habs $\cdot$ J. Schreiber $\cdot$ A. Henig

Max-Planck Institute of Quantum Optics, 85748 Garching, Germany

M. Hegelich

Los Alamos National Laboratories, Los Alamos, NM 87545, USA nostics in medicine. We want to obtain the X-rays by reflection of laser photons with energy $\hbar \omega$ from a relativistic electron mirror moving opposite with energy $E_{\mathrm{e}}=\gamma m_{\mathrm{e}} c^{2}$ using the Doppler effect to boost the photon energies to $4 \gamma^{2} \hbar \omega$ [1]. This general idea of a relativistic plasma mirror has existed for a long time [2, 3].

We describe the generation of an electron sheet, starting from an overdense plasma by driving the electrons out of an ultra-thin foil and then accelerating the sheet within a half-cycle of a laser pulse in vacuum to high energies [4]. A major issue is the coherent photon reflectivity off the electron sheet and choosing the right angle of the counterpropagating laser. While the technique of generating X-rays from a relativistic dense electron mirror with $\gamma \approx 5$ has recently been demonstrated experimentally by producing the electron sheet via a wake field, close to wave breaking, from an underdense plasma [5-7], the scaling properties and the process are very different from the mechanism discussed here.

One can also consider the thin dense relativistic electron sheet with its reflection of laser light from the viewpoint of a free-electron laser (FEL). If we replace in a classical X-FEL the magnetic undulator with period $\lambda_{\mathrm{u}}$ and undulator parameter $K=\lambda_{\mathrm{u}} e B /(2 \pi m c)$ by a laseran optical undulator-with $\lambda_{\mathrm{L}}$ and normalized vector potential $a_{\mathrm{L}}$ one can induce micro-bunches in the electron sheet, using then the coherent reflection of all electrons.

In this communication we discuss the production of the dense electron sheet, the vacuum acceleration, the conditions for optimum Lorentz energy boost of the reflected photons, and the reflectivity of the electron sheet in different orders of sophistication. 


\section{Generating the relativistic electron sheet by vacuum} acceleration

\subsection{Extraction of electron sheets from ultra-thin solid targets}

When the light pressure of a laser pulse irradiating a solid target pushes the electrons into the interior of the target, an electric field $E_{\mathrm{s}}$ arises due to charge separation. When the electrons of density $n_{\mathrm{e}}$ are displaced by the distance $d$, the Poisson equation yields $E_{\mathrm{s}}=e n_{\mathrm{e}} d / \varepsilon_{0}$. We normalize $E_{\mathrm{s}}$ to $E_{0}=c \omega_{\mathrm{L}} m_{\mathrm{e}} / e$ and the density $n_{\mathrm{e}}$ to the critical density $n_{\mathrm{cr}}=\omega_{\mathrm{L}}{ }^{2} \varepsilon_{0} m_{\mathrm{e}} / e^{2}$, where $c$ denotes the vacuum speed of light, $\omega_{\mathrm{L}}$ the carrier frequency of the laser pulse, $m_{\mathrm{e}}$ the electron mass, $e$ the electron charge, and $\varepsilon_{0}$ the permittivity of free space. Introducing $N=n_{\mathrm{e}} / n_{\mathrm{cr}}, a_{\mathrm{L}}=E_{\mathrm{L}} / E_{0}$, and the wave number $k_{\mathrm{L}}=\omega_{\mathrm{L}} / c$, we then obtain $E_{\mathrm{s}} / E_{0}=N k_{\mathrm{L}} d$. For break out (target thickness equals $d$ ), we require for the rectified laser field that $E_{\mathrm{L}}>E_{\mathrm{s}}$, or correspondingly,

$N k_{\mathrm{L}} d \leq a_{\mathrm{L}}$

A similar formula was recently derived in [8]. For our diamond-like carbon (DLC) foils, the density ratio amounts to $N=n_{\mathrm{e}} / n_{\mathrm{cr}}=660$ at $\lambda_{\mathrm{L}}=1 \mu \mathrm{m}$. Since at present the production of ultra-thin, hard DLC foils with good transparency is limited to the thickness $\left(d_{\min }\right)$ of $\sim 5 \mathrm{~nm}$, the normalized vector potential $a_{\mathrm{L}}$ has to exceed the value of 20 corresponding to a minimal focal intensity of $5 \times 10^{20} \mathrm{~W} / \mathrm{cm}^{2}$ to drive the electron sheet out of the foil. In addition, a high temporal contrast is required so that the laser prepulse does not ionize the foil in advance. The intensity profile has to be flattopped or super-Gaussian, too, to obtain a flat electron sheet. Here, the usage of plasma mirrors [9] is advantageous, but also the DLC target acts like a plasma mirror, because the low-intensity prepulse is transmitted.

\subsection{Laser vacuum acceleration of a single electron in a half-cycle to relativistic energies}

Next, we consider the vacuum acceleration of the freeelectron sheet to high energies. The basic properties can be obtained analytically by considering a single electron only. Following [10], we define $x$ as the propagation direction of the driver laser and assume a linearly polarized laser field, $E_{y}=a_{\mathrm{L}} \sin \tau$. Here, $a_{\mathrm{L}}=e A / m c^{2}, \gamma=E / m_{\mathrm{e}} c^{2}$ and $p=\beta \gamma$, where $A$ is the vector potential of the laser field, $E$ is the total energy of the electron, and $p$ its momentum normalized to $m_{\mathrm{e}} c$. The laboratory time is measured in units of $\omega_{\mathrm{L}}$ and the length in units of $k_{\mathrm{L}}$ leading to $\omega_{\mathrm{L}} t \Rightarrow t$ and $k_{\mathrm{L}} x \Rightarrow x$. We furthermore introduce $\tau=t-x$. We thus have $\gamma^{2}=1+p_{x}^{2}+p_{\top}^{2}$ and $\gamma-1=p_{x}=p_{\top}^{2} / 2$. The system has the invariants $\kappa:=\gamma-p_{x}=1$ and $p_{\top}-a_{\mathrm{L}}=0$. The relation between the derivatives is $\mathrm{d} / \mathrm{d} t=\mathrm{d} \tau / \mathrm{d} t \mathrm{~d} / \mathrm{d} \tau=(1-$ $\left.\beta_{x}\right)^{\cdot} \mathrm{d} / \mathrm{d} \tau=1 / \gamma \mathrm{d} / \mathrm{d} \tau$. We start with the transverse momentum $p_{y}(\tau=0)=0$ and have the equations $p_{y}=\gamma \mathrm{d} y / \mathrm{dt}=$ $\mathrm{d} y / \mathrm{d} \tau, p_{x}=\gamma \mathrm{d} x / \mathrm{d} t=\mathrm{d} x / \mathrm{d} \tau$, and $\mathrm{d} p_{y} / \mathrm{d} \tau=-E_{\mathrm{y}}$. Thus, we obtain $p_{y}=-a_{\mathrm{L}}(1-\cos \tau)$. Let us assume that the electron is initially at rest: $p_{x}=0$. Then, $E_{\text {kin }} / m_{\mathrm{e}} c^{2}=\gamma-1=$ $p_{x}=p_{\top}^{2} / 2=1 / 2 a_{\mathrm{L}}{ }^{2}(1-\cos \tau)^{2}$ increases from 0 with $(1-\cos \tau)^{2}$ and reaches $2 a_{\mathrm{L}}^{2} m_{\mathrm{e}} c^{2}$ for $\tau=\pi$. The laser field drops back to zero for $\tau=\pi$. The electron trajectory is described by $y(\tau)=-a_{\mathrm{L}}(\tau-\sin \tau)$ resulting from $\mathrm{d} y / \mathrm{d} \tau=p_{y}$ and $x(\tau)=a_{\mathrm{L}}{ }^{2} / 2\left[3 / 2 \tau-2 \sin (\tau)+\frac{1}{4} \sin (2 \tau)\right]$ resulting from $\mathrm{d} x / \mathrm{d} \tau=p_{x}$. Replacing $t(\tau)=\tau+x(\tau)$ and starting from an electron at rest for $t=0$, we reach the maximum energy after the rather long time $t_{1}=(3 \pi / 4) a_{\mathrm{L}}{ }^{2}$, because the electron surfs with the wave. We obtain for $\tau=\pi$

$p_{y}=-2 a_{\mathrm{L}}, \quad p_{x}=2 a_{\mathrm{L}}^{2}, \quad \gamma=1+2 a_{\mathrm{L}}^{2}$.

Since we finally want to have a minimum energy spread $\Delta \gamma / \gamma$, we should operate with an $a_{\mathrm{L}}$ where the wanted energy saturates at $t_{1}$. The angle $\theta$ of the electron with respect to the driver laser is given by

$\tan \theta=p_{y} / p_{x}=-2 /\left[a_{\mathrm{L}}(1-\cos \tau)\right]$,

where $\tan \theta=-1 / a_{\mathrm{L}}$ for $\tau=\pi$ and varies little with $\tau$ in this time region. The absolute value of $\theta$ is very large compared to $1 / \gamma=1 /\left(1+2 a_{\mathrm{L}}^{2}\right)$. This will be of importance when we discuss Lorentz boosts of reflected photons.

\subsection{Laser acceleration of a dense electron sheet in vacuum}

Particle-in-cell (PIC) simulations show that a dense electron sheet including the space-charge Coulomb repulsion of the electrons increases the sheet thickness and reduces the electron density [4]. A laser pulse super-Gaussian in space and time leads to a much flatter electron sheet compared to a Gaussian pulse. However, the main characteristics of the acceleration with the transverse component and the long acceleration time due to slippage of the electrons with respect to the laser pulse stay the same as for the single-electron case discussed above.

\section{Reflectivity from dense electron sheets}

3.1 Relativistic photon reflection from a flat electron sheet

For the Lorentz transformation of photons from the laboratory frame to the inner reference frame of the electrons, the relativistic Doppler shift formulas are [11]

$\sin \theta^{\prime} /\left[\gamma\left(\cos \theta^{\prime}+\beta\right)\right]=\tan \theta$,

$\omega^{\prime}=\omega /[\gamma(1-\beta \cos \theta)]$, 


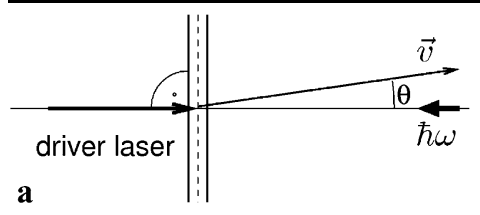

$\mathbf{a}$

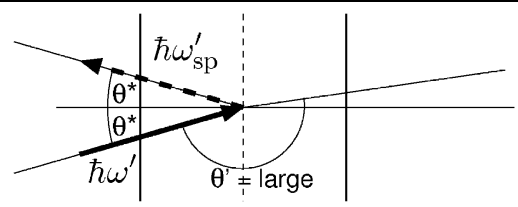

mirror
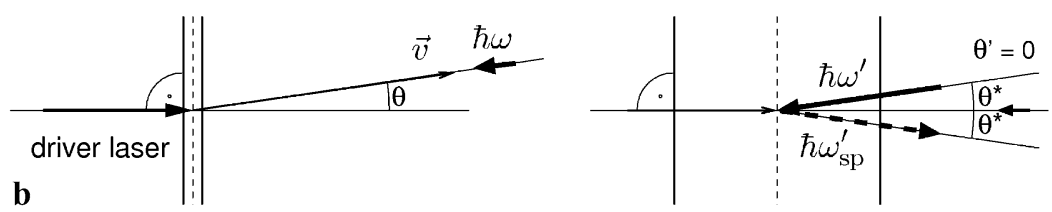
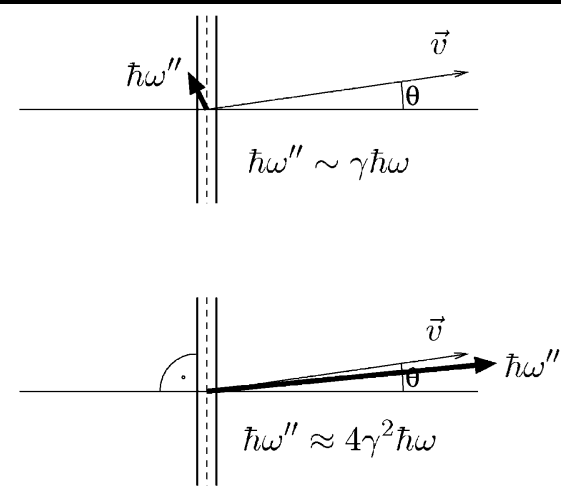

Fig. 1 The Doppler transformation yields rather different results depending on whether the 'production' photons $\hbar \omega$ are injected (a) opposite to the driver-laser direction or (b) opposite to the electron velocity $\boldsymbol{v}$, resulting in very different energies $\hbar \omega^{\prime \prime}$ and directions of the re- emitted photons in the laboratory system. The change in direction of the photons $\hbar \omega^{\prime}$ in the inner rest frame is at first unexpected. The dashed photon arrows $\hbar \omega_{\mathrm{sp}}^{\prime}$ correspond to reflected photons where $\theta$ and $\theta^{\prime}$ are the angles relative to the velocity $v=\beta c$, and $\omega, \omega^{\prime}$ are the photon frequencies. The inverse equations are obtained by changing the sign of $v$ and changing the dashed and undashed quantities.

We have obtained the angle $\theta$ of the electron and its energy after vacuum acceleration relative to the direction of the driver laser from the relations $\tan \theta=-1 / a_{\mathrm{L}}$ and $\gamma=1+2 a_{\mathrm{L}}{ }^{2}$ at $\tau=\pi$. If we inject a photon opposite to the driver laser, $\cos \theta$ is 1 and $\omega^{\prime}=2 \gamma \omega$, but $\theta^{\prime}$ in the electron rest frame is approximately $180^{\circ}$ with respect to the electron direction, because $1 / \gamma \ll|\theta|$. If we now perform a mirroring at the foil surface with entrance angle = exit angle, the mirrored photon again runs opposite to the electron direction. If we perform the Lorentz transformation back into the laboratory system, the photon energy is around $\gamma \hbar \omega$ and is not boosted by $4 \gamma^{2}$. These steps of operation are schematically shown in Fig. 1.

If, on the other hand, we inject the photons with an angle much smaller than $1 / \gamma$ opposite to the direction of the electrons with $\theta \approx 0$, the energy is again boosted by $2 \gamma$ but now $\theta^{\prime}=0$. If we now perform the mirroring with respect to the foil, we obtain the angle $2 / a_{\mathrm{L}}$. This photon after transformation to the laboratory system obtains a second boost by $2 \gamma$ and is emitted into a cone of very small apex angle whose axis coincides with the electron velocity. Thus, the total boost is $4 \gamma^{2}$.

Injecting the production laser counter-propagating to the electron beam results in a $180^{\circ}$ turn of the production-laser photon direction in the inner rest frame of the electrons, which finally leads to the $4 \gamma^{2}$ Doppler boost in energy. In the other case when the production laser counter-propagates the driver laser the energy gain due to Doppler boost merely amounts to $\gamma$. For a fixed electron direction, the productionlaser angle is limited to a very small angular spread $<1 / \gamma$. If the photons of maximal energy are filtered out, the system will select the proper angles for maximum energies from the different focused angles of the production laser. The issue of a transverse momentum component has not been addressed in former treatments [4] of dense electron sheets accelerated in vacuum and their use as relativistic mirrors.

\subsection{Idealized coherent reflectivity for an electron sheet with constant thickness $d$, constant electron density $n_{\mathrm{e}}$, and monoenergetic electrons characterized by $\gamma$}

Here, we first want to derive the coherent reflectivity for photons from an electron sheet for the idealized case of constant thickness $d$, constant electron density $n_{\mathrm{e}}$, and constant kinetic energy characterized by $\gamma$. Later we will discuss a more realistic situation.

If the electron sheet has the thickness $d_{\mathrm{i}}=\gamma d$ in the inner rest frame determined by both the front and backward surface layers, the number of coherently reflected photons in the rest frame is $R \propto\left(n_{\mathrm{e}} \sin 2 \pi d_{\mathrm{i}} / \lambda_{\mathrm{i}}\right)^{2}$, showing constructive and destructive interference. The contribution of each layer with thickness $\lambda_{\mathrm{I}}=\lambda /(2 \gamma)$ cancels. Only the incremental part $\Delta d=\gamma d-m \lambda_{\mathrm{i}}$ contributes, where the integer $m$ is determined by $|\Delta d| \leq \lambda_{\mathrm{i}}$. Different regions may have slightly different thicknesses $d$ and the scattered amplitudes will interfere and a smaller value than just due to the averaged $\Delta d$ occurs.

\subsection{Optical trapping and compression of an electron sheet} by counter-propagating lasers

We want to study the trapping of the electron sheet between the driver laser and the production laser. Since the frequency of the driver laser in the inner reference frame of the electron sheet is reduced to $\omega_{\text {low }}=\omega^{\prime} /(2 \gamma)$, while the frequency of the production laser is increased to $\omega_{\text {high }}=2 \omega \gamma$, we study the trapping between a low- and a high-frequency laser, which is quite different from the trapping between two lasers 
with the same frequency [12]. The force of the laser with the low frequency, $\omega_{\text {low }}$, will vary slowly with $\sin ^{2}\left(\omega_{\text {low }} t\right)$, while for the laser with the high frequency, $\omega_{\text {high }}$, the variation $\sin ^{2}\left(\omega_{\text {high }} t\right)$ can be replaced by $1 / 2$. Therefore, the thickness of the foil will vary with $d(t)=d_{\mathrm{i}}-d_{1} \sin ^{2}\left(\omega_{\text {low }} t\right)$ with $d_{1}<d_{\mathrm{i}}$, whereby $d_{1}$ is accessible by numerical simulations.

The surfaces of the electron sheet are defined by the laser potentials and thus the local surface fluctuations around the globally defined surfaces can be neglected, similar to the case of high harmonics [13].

Increasing the electron density $n_{\mathrm{e}}$ by the driving laser using intensities beyond $10^{22} \mathrm{~W} / \mathrm{cm}^{2}$, a dominant transfer of energy to radiation and not to the electrons was observed numerically [14]. In this way fast laser cooling may become possible.

\subsection{Allowable energy spread of the electron sheet}

Due to the uncertainty relation, the emission time of Compton backscattering is so fast that no smearing of coherence by electron motion occurs.

However, the formulas for the reflectivity with constant $\gamma$ show that the energy spread $\Delta \gamma / \gamma$ has to fulfill the relation $\Delta \gamma / \gamma \leq \pi /\left(32 \gamma^{2} k_{\mathrm{L}} d\right)$. Since this requirement can only be fulfilled for smaller $\gamma$ values, it strongly limits the reflectivity and the extension to higher X-ray energies. On the other hand, the FEL-like micro-bunching overcomes this limitation due to the rather large Pierce parameter.

\subsection{FEL-like micro-bunching of the electron sheet}

The main idea of the free-electron laser (FEL) is that the produced high-energy photon beam with wavelength $\lambda_{x}$ acts back by dispersion on to the electron ensemble and induces an equally spaced micro-bunching of the electrons with distance $\lambda_{x}$ in such a way that all backscattering amplitudes of the micro-bunches add coherently. For the sheets of high electron density and very small thickness, we start out with one micro-bunch only when the X-ray energies are small. However, at higher $\gamma$ energies or shorter wavelengths, we would only use the incremental thickness $\Delta d$, while the other $m$ oscillations of the reflectivity would interfere destructively. Therefore, it is advantageous to use the microbunching here also.

The Pierce or FEL parameter, $\rho_{1 \mathrm{~d}}$, is used to describe the FEL in the one-dimensional idealized case

$\rho_{1 \mathrm{~d}}=1 / 2 \gamma\left[I / I_{\mathrm{A}}\left(\lambda_{\mathrm{u}} A_{\mathrm{u}} / 2 \pi \sigma_{x}\right)^{2}\right]^{1 / 3}$.

Here, $I$ is the beam current, $I_{\mathrm{A}}=17 \mathrm{kA}$ is the Alfvén current, $\sigma_{x}$ is the beam size, $A_{\mathrm{u}}=a_{\mathrm{u}}\left[J_{0}(\eta)-J_{1}(\eta)\right]$ (planar undulator), and $K$ is the undulator parameter, where $a_{\mathrm{u}}{ }^{2}=$
$K^{2} / 2, \eta=a_{\mathrm{u}}^{2} /\left(2\left(1+a_{\mathrm{u}}^{2}\right)\right)$, and $J$ are Bessel functions. $\lambda_{\mathrm{u}}$ is the wavelength of the undulator.

The Pierce parameter gives the limit for the maximum allowable energy spread

$\Delta \gamma / \gamma \leq \rho_{1 \mathrm{~d}}$.

The ideal gain length for micro-bunching is

$L_{\text {gain, ideal }}=\lambda_{\mathrm{u}} / 4 \pi \sqrt{3} \rho_{1 \mathrm{~d}}$.

If a sufficient intensity of the X-ray photons above the noise is reached, about eight gain lengths are required to reach saturation of the micro-bunching. In our case without any micro-bunching, a high X-ray intensity is reflected off the strongly enhanced density of the sheet area close to the driver laser.

In contrast to classical FELs with a magnetic undulator, we use the production laser as an optical undulator. The wavelength of the optical undulator $\lambda_{\mathrm{u}}$ of about $1 \mu \mathrm{m}$ is typically $10^{4}$ times smaller than that for magnetic undulators, which have minimum undulator periods of $\sim 10 \mathrm{~mm}$. This small undulator wavelength $\lambda_{\mathrm{u}}$ results in a strong reduction of the Pierce parameter $\propto \lambda_{\mathrm{u}}^{-2 / 3}$. On the other hand, we use much smaller electron energies characterized by $\gamma$ to achieve the same final wavelength $\lambda_{\mathrm{u}} /\left(4 \gamma^{2}\right)$. This increases the Pierce parameter by $\lambda_{u}^{1 / 2}$. Thus, in total, a weak $\lambda_{\mathrm{u}-1 / 6}$ dependence remains. Besides the increase in the Pierce parameter by the significantly smaller $\sigma_{x}$ values, a further strong increase is obtained by the much larger currents $I$ of the electron sheet. Due to the laser compression we can reach currents with the electron sheet which are much larger than the Alfvén limit $17 \mathrm{kA} \gamma \beta$. In the inner rest frame of the electron sheet, the laser trapping prevents the longitudinal Coulomb explosion. The transverse Coulomb explosion is not effective due to the large transverse extension of the electron sheet. Thus, much larger stable electron densities are obtained, which after Lorentz transformation into the laboratory frame allow currents much beyond the Alfvén limit.

Since the gain length is proportional to the undulator period, we obtain a saturation length of the FEL of about $25 \mu \mathrm{m}$. Thus, we can use the large electron number of the electron sheet completely and the reflectivity $R$ including micro-bunching is given by $R \propto n_{\mathrm{e}}^{2} d^{2}$. The micro-bunching seems to be unavoidable, although it has to be studied in detail how strong it is as a function of photon energy and how big the change in reflectivity will be. The larger Pierce parameter furthermore reduces the requirement on the energy spread with $\Delta \gamma / \gamma \leq \rho_{1 \mathrm{~d}}$. In this way it seems possible to extend the energy range of the photon beams into the $\mathrm{MeV}$ range. For 6-GeV electrons the energy loss when radiating a $1-\mathrm{GeV}$ photon just stays within the $20 \%$ allowed energy spread of the electron sheet. This allowable energy spread is one of the limits for the maximum achievable photon energy. 
Table 1 Parameters for the

comparison between table-top

X-ray FEL [15] and the X-ray

source from a dense relativistic mirror with the same X-ray

energy and for $1-\mathrm{GeV}$ photons
TT-XFEL

\begin{tabular}{llll}
\hline Current & $160 \mathrm{kA}$ & $10^{6} \mathrm{kA}$ & $10^{14} \mathrm{kA}$ \\
Electrons/bunch & $6 \times 10^{9}$ & $3 \times 10^{10}$ & $6 \times 10^{11}$ \\
Beam size & $30 \mu \mathrm{m}$ & $1 \mu \mathrm{m}$ & $1 \mu \mathrm{m}$ \\
Energy & $1.7 \mathrm{GeV}$ & $17.5 \mathrm{MeV}$ & $6.2 \mathrm{GeV}$ \\
Energy spread & $0.1 \%$ & $3 \%$ & $3 \%$ \\
Period & $5 \mathrm{~mm}$ & $1 \mu \mathrm{m}$ & $1 \mu \mathrm{m}$ \\
Final wavelength & $0.25 \mathrm{~nm}$ & $0.25 \mathrm{~nm}$ & $1.2 \mathrm{fm}$ \\
Final energy & $5 \mathrm{keV}$ & $5 \mathrm{keV}$ & $1 \mathrm{GeV}$ \\
Pierce parameter & 0.0015 & 0.2 & 0.2 \\
Saturation length & $5 \mathrm{~m}$ & $25 \mu \mathrm{m}$ & $25 \mu \mathrm{m}$ \\
Photons/bunch & $8 \times 10^{11}$ & $10^{13}$ & $10^{12}$ \\
Photon bunch duration & $5 \mathrm{fs}$ & $\sim 4 \mathrm{as}$ & $\sim 0.2 \mathrm{zs}$ \\
\hline
\end{tabular}

In Table 1 we compare the new method to produce X-rays with the relativistic electron sheet to an envisaged table-top X-FEL (TT-XFEL) [15]. We also show the far extension to $1-\mathrm{GeV}$ photon beams.

\section{Outlook}

Besides the photon intensities, the emittances and peak brilliances are also important. Here, we may use the parabolically shaped electron sheet to focus the $\gamma$-beams and obtain diffraction-limited $\gamma$-ray beams $[6,16]$. With the numbers from Table 1 we can estimate the peak brilliances $P$ with $\mathrm{BW}=$ 'bandwidth' of the beams. We obtain $P(5 \mathrm{keV})=$ $2 \times 10^{46}$ photons $/ \mathrm{s} \cdot(\mathrm{mm} \mathrm{mrad})^{2} \cdot 30 \% \mathrm{BW}$ and $P(1 \mathrm{GeV})=$ $7 \times 10^{59}$ photons $/ \mathrm{s} \cdot(\mathrm{mm} \mathrm{mrad})^{2} \cdot 30 \% \mathrm{BW}$. In contrast to former definitions of the peak brilliance, we do not refer to a bandwidth of $0.1 \%$, but to the typical bandwidth of $30 \%$, which we want to use in experiments. If one would reduce the bandwidth by a factor of 300 with a monochromator to $0.1 \%$, the pulse duration would also be increased by a factor of 300 and the peak brilliance would be reduced by a factor of $9 \times 10^{4}$. However, since we want to focus on experiments with very short pulse duration, it is not reasonable to reduce the bandwidth to $0.1 \%$ when characterizing our beams. Still, the predicted peak brilliances are much larger than the brilliances of the planned X-FELs of $10^{34}$ photons $/ \mathrm{s} \cdot(\mathrm{mm} \mathrm{mrad})^{2} \cdot 0.1 \% \mathrm{BW}$. Besides the peak brilliance, other reference values like the total number of photons per bunch are important to characterize the beams.

If we assume that we can operate a $\gamma-\gamma$ collider with $f_{0}=1 \mathrm{~Hz}$ and that we can focus our intensities to $\sigma_{y}=\sigma_{z}=$ $1 \mathrm{fm}$ by the parabolically shaped electron sheet mirrors, we obtain a very large luminosity, $L=1 / 2 \pi N_{1} N_{2} /\left(\sigma_{y} \sigma_{z}\right)=$ $2 \times 10^{50} /\left(\mathrm{cm}^{2} \mathrm{~s}\right)$, for photon numbers $N_{1}=N_{2}=10^{12}$ per bunch.
With an expected hadronic cross section of $\sigma_{\gamma \gamma}=$ 1 pbarn, we would obtain a very large event rate, $\dot{N}=$ $\sigma_{\gamma \gamma} L=2 \times 10^{20} \mathrm{~s}^{-1}$, which shows that all the energy would be accumulated in a rather small volume with the very high energy density of $\sim 10^{12} \mathrm{GeV} / \mathrm{fm}^{3}$. Certainly we will have much larger beam dimensions in the beginning and we will have to align the beams with respect to each other. Also, problems with the pointing stability will reduce the event rates or the energy density.

The final limits for the $\gamma$-laser are determined by the size of the Pierce parameter and if radiation cooling of the compressed electron sheet allows even higher beam currents with lower energy spread.

With such brilliant, intense $\mathrm{MeV} \gamma$-beams one could study the excitation of nuclei or production of positrons. If it would even be possible to reach energies of $1 \mathrm{GeV}$, new horizons would open up for ELI [17], the European Extreme-Light-Infrastructure aiming at providing lasers in the Exawatt range.

Thus, this photon beam would have a significant admixture - about $0.3 \%$ — of vector mesons — like $\rho$ mesons [18]and one could even think of a $\gamma-\gamma$ collider with a hadronic cross section of $0.7 \mu$ barn [19]. One could regard the $\gamma-\gamma$ collider as a virtual vector meson-vector meson collider, although real mesons have a half-life of $10^{-20}$ to $10^{-24} \mathrm{~s}$. In optimized collisions a large amount of energy of about $10^{12} \mathrm{GeV}$ could be deposited without spin into a very small volume, reaching energy densities of $10^{12} \mathrm{GeV} / \mathrm{fm}^{3}$, which is large compared to the energy density of the quarkgluon phase transition of about $3 \mathrm{GeV} / \mathrm{fm}^{3}$. If we write the energy density as $\varepsilon_{\mathrm{QG}}\left(T / T_{\mathrm{c}}\right)^{4}$ and use $T_{\mathrm{c}}=170 \mathrm{MeV}$ and $\varepsilon_{\mathrm{QG}}=3 \mathrm{GeV} / \mathrm{fm}^{3}$ from the quark-gluon phase transition, we reach a temperature of about $170 \mathrm{GeV}$ in the region of the electro-weak phase transition $\left(m_{\mathrm{Z}}=91 \mathrm{GeV}\right.$, $\left.m_{\mathrm{W}}=80 \mathrm{GeV}\right)$. Possibly also the Higgs boson and a neutralino SUSY candidate for dark matter [20] come into 
reach. Thus, the collider could be viewed as a factory for many exotic particles like Higgs bosons, dark matter or the quark-gluon plasma. The energy density is large compared to the energy densities reached in the $2.7-\mathrm{TeV} \mathrm{Pb}-\mathrm{Pb}$ collider (LHC). The energy density of $10^{12} \mathrm{GeV} / \mathrm{fm}^{3}$ or a temperature of $170 \mathrm{GeV}$ corresponds to the situation $10^{-9} \mathrm{~s}$ after the big bang and many questions of high-energy physics could be studied.

Presently, large PIC simulations have been started with smaller $\gamma$ values to verify the new concept within reasonable computing times. Also, non-linear FEL theory will be added.

Acknowledgements We acknowledge many useful discussions with J. Meyer-ter-Vehn, H.C. Wu, H. Ruhl, F. Grüner, R. Hörlein, B.J. Albright, and L. Yin.

The work was supported by the Deutsche Forschungsgemeinschaft through the DFG Cluster of Excellence 'Munich Centre for Advanced Photonics' (MAP) and Transregio TR18.

Open Access This article is distributed under the terms of the Creative Commons Attribution Noncommercial License which permits any noncommercial use, distribution, and reproduction in any medium, provided the original author(s) and source are credited.

\section{References}

1. A. Einstein, Ann. Phys. 17, 891 (1905)

2. S.V. Bulanov, N.M. Naumova, F. Pegoraro, Phys. Plasmas 1, 745 (1994)

3. J.M.J. Madey, J. Appl. Phys. 42, 1906 (1971)
4. V.V. Kulagin, V.A. Cherepenin, M.S. Hur, H. Suk, Phys. Rev. Lett. 99, 124801 (2007)

5. A.S. Pirozhkov, J. Ma, M. Kando, T.Z. Esirkepov, Y. Fukuda, L.-M. Chen, I. Daito, K. Ogura, T. Homma, Y. Hayashi, H. Kotaki, A. Sagisaka, M. Mori, J.K. Koga, T. Kawachi et al., Phys. Plasmas 14, 123106 (2007)

6. S.V. Bulanov, T. Esirkepov, T. Tajima, Phys. Rev. Lett. 91, 085001 (2003)

7. A.V. Panchenko, T.Z. Esirkepov, A.S. Pirozhkov, M. Kando, F.F. Kamenets, S.V. Bulanov, Interaction of electromagnetic waves with caustics in plasma physics. Phys. Plasmas (2008, submitted)

8. O. Klimo, J. Psikal, J. Limpouch, V.T. Tikhonchuk, Phys. Rev. St AB 11, 031301 (2008)

9. R. Hörlein, B. Dromey, D. Adams, Y. Nomura, S. Kar, K. Markey, P. Foster, D. Neely, F. Krausz, G.D. Tsakiris, M. Zepf, New J. Phys. 10, 083002 (2008)

10. J. Meyer-ter-Vehn, A. Puhkov, Z.M. Sheng, Relativistic laser plasma interactions, in Proc. 30th Course Int. School of Quantum Electronics, ed. by D. Batani et al., Erice, Italy, 8-14 July 2000 (Kluwer Academic/Plenum, New York, 2001), pp. 167-192

11. J.H. Jackson, Classical Electrodynamics (Wiley, New York, 1972)

12. B. Shen, J. Meyer-ter-Vehn, Phys. Plasmas 8, 1003 (2001)

13. T. Baeva, High harmonics generation from relativistic plasma. Thesis, Heinrich-Heine University (2008)

14. N. Naumova et al., Hole boring in a DT pellet and fast ion ignition with ultra-intense laser pulses. Phys. Rev. Lett. (2008, submitted)

15. F. Grüner, S. Becker, U. Schramm, T. Eichner, M. Fuchs, R. Weingartner, D. Habs, J. Meyer-ter-Vehn, M. Geissler, M. Ferrario, L. Serafini, B. van der Geer, H. Backe, W. Lauth, S. Reiche, Appl. Phys. B 86, 431 (2007)

16. S. Gordienko, A. Pukhov, O. Shorokhov, T. Baeva, Phys. Rev. Lett. 94, 103903 (2005)

17. http://www.extreme-light-infrastructure.eu/

18. G. Baur, K. Hencken, D. Trautmann, S. Sadovsky, Y. Kharlov, Phys. Rep. 364, 359 (2002)

19. Particle Data Group, Phys. Lett. B 667, 363 (2008)

20. D. Elsässer, K. Mannheim, Phys. Rev. Lett. 94, 171302 (2005) 